\title{
Formas Hepatosplênicas da Esquistossomose Mansônica no Interior do Nordeste do Brasil
}

\author{
Hepatosplenic Forms of Schistosomiasis Mansoni in the Interior of \\ Northeastern Brazil
}

\author{
Frederico S. Barbosa ${ }^{1}$ \\ José Felipe Gonçalves ${ }^{1}$ \\ Marcelo C. V. de Melo
}

\section{BARBOSA, F. S.; GONÇALVES, J. F. \& MELO, M. C. V. Hepatosplenic Forms of Schistosomiasis}

Mansoni in the Interior of Northeastern Brazil. Cad. Saúde Públ., Rio de Janeiro, 11 (2): 325-331, Apr/Jun, 1995.

Field studies on the morbidity of schistosomiasis mansoni were carried out in some rural endemic areas of Northeastern Brazil during the first semester of 1991. These cross-sectional studies were limited to the assessment of the gradient of clinical forms of the disease. The entire population present in a house-to-house survey was examined. Results have shown a reduction in the prevalence rates of the more severe form of schistosomiasis, the hepatosplenic form, when compared with previous similar studies made in the same areas during the 1960s and 1970s. Changes in prevalence rates of the severe clinical forms from the younger to the older age groups were observed.

Key words: Schistosomiasis; Morbidity; Epidemiology

\section{INTRODUÇÃO}

Estudos de campo sobre as formas hepatosplênicas (H.E.) da esquistossomose no Brasil são conhecidos desde a década de 50 (Pessoa \& Barros, 1953; Pessoa et al., 1955; Pessoa \& Amorim, 1957; Brener \& Mourão, 1956). Com estes dados e os muitos estudos clínicos realizados no país firmou-se a convicção de que a forma hepatosplênica representa o indicador de maior gravidade da doença esquistossomótica em populações de algumas regiões do país, particularmente

\footnotetext{
'Centro de Pesquisas Aggeu Magalhães, Fundação Oswaldo Cruz. Campus da Universidade Federal de Pernambuco, Engenho de Meio, Recife, PE, 50670-240, Brasil.

${ }^{2}$ Hospital das Clínicas, Universidade Federal de Pernambuco. Campus da Universidade Federal de Pernambuco, Engenho de Meio, Recife, PE, 50670-240, Brasil.
}

naquelas áreas onde a endemia mostrava maior prevalência e piores condições sócioeconômicas (Kloetzel, 1962; Barbosa, 1965; Katz \& Brener, 1966; Kloetzel, 1967). A irreversibilidade, tanto das formas clínicas graves como das lesões patológicas, era geralmente admitida.

A introdução da oxamniquine - o medicamento mais utilizado no Brasil - no arsenal terapêutico da esquistossomose trouxe inúmeras esperanças para o controle da endemia e, particularmente, para a reversão da forma hepatosplênica e conseqüente redução da morbidade em termos de população.

O objetivo do presente trabalho é conhecer a prevalência das formas hepatosplênicas em áreas endêmicas limitadas de três estados brasileiros onde estudos semelhantes foram realizados; nas décadas de 60/70. Os dois cortes transversais foram realizados, o primeiro antes e o segundo depois das ações terapêuticas desenvolvidas no nordeste brasileiro, pelo Programa Especial de Controle da Esquistossomose (PECE). 


\section{MÉTODOS}

Neste trabalho foram utilizados dados secundários cedidos pela antiga Superintendência de Campanhas de Saúde Pública (Sucam), dados publicados ou inéditos, e dados primários coletados no primeiro semestre de 1991, nas localidades indicadas a seguir. No Rio Grande do Norte: sítios Jardim, São José Tapera, Olho Dágua e Maceió, área rural do município de Pureza. Em Pernambuco: engenhos Boa Vista e Pau do óleo, município de Catende. Em Alagoas: Vila Anel, município de Viçosa e Usina Capricho, município de Cajueiro. Detalhes sobre as áreas trabalhadas encontram-se em publicações anteriores (Barbosa et al., 1970a; Barbosa et al., 1970b; Costa \& Barbosa, 1980).

Exames clínicos e exames parasitológicos das fezes foram realizados em toda a população pre- sente no momento da visita domiciliar. Os exames clínicos limitaram-se, como nos exames anteriores, às técnicas semiológicas clássicas, não armadas, não tendo havido, obviamente, preocupação na utilização de técnicas mais complexas e exames clínicos mais detalhados. Para os exames clínicos, utilizou-se a classificação de Pessoa \& Barros (1953) modificada por Barbosa \& Voss (1969). Foi feito um único exame de fezes, por sedimentação simples, para cada pessoa incluída no estudo.

\section{RESULTADOS}

Os resultados obtidos neste trabalho, em comparação com dados anteriormente observados, são apresentados a seguir de acordo com os estados da federação onde foram realizados.

\section{Estado de Alagoas}

TABELA 1. Dados Agregados, em Nível de Município, Sobre a Prevalência da Infecção Esquistossomótica e a Cobertura Medicamentosa, Alagoas

\begin{tabular}{lccccccc}
\hline \hline Município & 1977 & 1978 & 1979 & 1980 & 1981 & 1982 & 1983 \\
\hline - Cajueiro & & & & & & & \\
$\quad$ Prevalência \% & 74,3 & - & 36,3 & - & 38,7 & - & 16,1 \\
$\quad$ \% População tratada & - & 67,9 & - & 49,4 & 67,8 & - & 80,2 \\
- Viçosa & & & & & & & 19,8 \\
$\quad$ Prevalência \% & 66,5 & - & 38,2 & - & 32,2 & - & 64,6 \\
$\quad$ \% População tratada & - & 62,5 & - & 43,8 & 61,1 & - & \\
\hline \hline
\end{tabular}

Fonte: Fundação Nacional de Saúde, Diretoria Regional de Alagoas.

TABELA 2. Prevalência da Infecção e da Forma Hepatosplênica da Esquistossomose, por Grupo Etário e Sexo, na Usina Capricho, Cajueiro, Alagoas, em 1967 e 1991

\begin{tabular}{|c|c|c|c|c|c|c|c|c|c|c|c|c|c|c|c|c|}
\hline \multirow{2}{*}{$\begin{array}{l}\text { Ano Prevalência } \\
\text { Forma H.E. }\end{array}$} & & \multicolumn{3}{|c|}{ Total Geral } & \multicolumn{3}{|c|}{$<9$} & \multicolumn{3}{|c|}{$10-19$} & \multicolumn{3}{|c|}{$20-39$} & \multicolumn{3}{|c|}{$>40$} \\
\hline & & $\mathrm{T}$ & M & F & $\mathrm{T}$ & $\mathrm{M}$ & $\mathrm{F}$ & $\mathrm{T}$ & $\mathrm{M}$ & F & $\mathrm{T}$ & $\mathrm{M}$ & F & $\mathrm{T}$ & $\mathrm{M}$ & F \\
\hline \multicolumn{17}{|l|}{$1967^{\mathrm{A}}$} \\
\hline População examinada & & 452 & 203 & 249 & 215 & 104 & 111 & 103 & 47 & 56 & 99 & 34 & 65 & 35 & 18 & 17 \\
\hline \multirow[t]{2}{*}{ Forma H.E. } & $11^{\circ}$ & 7 & 5 & 2 & 0 & 0 & 0 & 4 & 4 & 0 & 2 & 1 & 1 & 1 & 0 & 1 \\
\hline & $\%$ & 1,5 & 2,5 & 0,8 & - & - & - & 3,9 & 8,5 & 0 & 2,0 & 2,9 & 1,5 & 2,9 & - & 5,9 \\
\hline \multicolumn{17}{|l|}{$1991^{\mathrm{B}}$} \\
\hline População examinada & & 488 & 250 & 238 & 139 & 84 & 55 & 125 & 64 & 61 & 134 & 60 & 74 & 90 & 42 & 48 \\
\hline \multirow[t]{2}{*}{ Forma H.E. } & $\mathrm{n}^{\circ}$ & 5 & 1 & 4 & 0 & 0 & 0 & 0 & 0 & 0 & 2 & 1 & 1 & 2 & 0 & $2^{C}$ \\
\hline & $\%$ & 1,0 & 0,4 & 1,7 & - & - & - & - & - & - & 1,5 & 1,7 & 1,4 & 2,2 & - & 4,2 \\
\hline Prevalência & $\%$ & 22,5 & 27,2 & 17,6 & 21,6 & 26,2 & 17,0 & 30,4 & 34,3 & 26,2 & 23,9 & 33,8 & 16,2 & 11,1 & 9,5 & 12,5 \\
\hline
\end{tabular}


TABELA 3. Prevalência da Infecção e da Forma Hepatosplênica da Esquistossomose, por Grupo Etário e Sexo, Zona Rural de Viçosa, Alagoas, 1966 e 1991

\begin{tabular}{|c|c|c|c|c|c|c|c|c|c|c|c|c|c|c|c|c|}
\hline \multirow{2}{*}{$\begin{array}{l}\text { Ano Prevalência } \\
\text { Forma H.E. }\end{array}$} & & \multicolumn{3}{|c|}{ Total Geral } & \multicolumn{3}{|c|}{$<9$} & \multicolumn{3}{|c|}{$10-19$} & \multicolumn{3}{|c|}{$20-29$} & \multicolumn{3}{|c|}{$>40$} \\
\hline & & $\mathrm{T}$ & $\mathrm{M}$ & $\mathrm{F}$ & $\mathrm{T}$ & M & $\mathrm{F}$ & $\mathrm{T}$ & M & $\mathrm{F}$ & $\mathrm{T}$ & M & $\mathrm{F}$ & $\mathrm{T}$ & M & F \\
\hline \multicolumn{17}{|l|}{$1966^{\mathrm{A}}$} \\
\hline População Examinada & & 906 & 395 & 511 & 201 & 143 & 158 & 223 & 90 & 133 & 234 & 92 & 142 & 148 & 70 & 78 \\
\hline \multirow[t]{2}{*}{ Forma H.E. } & $\mathrm{n}^{\circ}$ & 32 & 18 & 14 & 1 & 1 & 0 & 9 & 6 & 3 & 8 & 5 & 3 & 14 & 6 & 8 \\
\hline & $\%$ & 3,5 & 4,6 & 2,7 & 0,5 & 0,7 & - & 4,0 & 6,7 & 2,6 & 3,4 & 5,4 & 2,1 & 9,5 & 8,6 & 10,3 \\
\hline $\begin{array}{l}\text { Prevalência } \\
1991^{\text {B }}\end{array}$ & $\%$ & 72 & 72 & 72 & 36 & 35 & 36 & 90 & 89 & 91 & 92 & 95 & 90 & 80 & 83 & 77 \\
\hline População examinada & & 488 & 203 & 285 & 132 & 68 & 64 & 159 & 67 & 92 & 85 & 22 & 63 & 112 & 46 & 66 \\
\hline \multirow[t]{2}{*}{ Forma H.E. } & $\mathrm{n}^{\circ}$ & 5 & 2 & 3 & 0 & 0 & 0 & 0 & 0 & 0 & 2 & lc & 1 & 3 & 1 & $2^{\mathrm{C}}$ \\
\hline & $\%$ & 1,0 & 0,9 & 1,1 & - & - & - & - & - & - & 2,4 & 4,5 & 1,6 & 2,7 & 2,2 & 3,0 \\
\hline Prevalência & $\%$ & 3,5 & 3,0 & 3,9 & 2,3 & 2,9 & 1,6 & 1,9 & 1,5 & 2,3 & 4,7 & 9,1 & 3,2 & 6,3 & 2,2 & 9,1 \\
\hline
\end{tabular}

A. Fonte: Barbosa et al., 1970a, dados agregados para área rural de Viçosa (Alagoas).

B. Dados primários para a localidade de Anel (Viçosa, Alagoas).

C. Inclui um esplenectomisado. Os dois casos foram operados em Maceió, nos anos de 1987 e 1989.

Para o município de Viçosa (Tabela 3) os dados de 1966 (Barbosa et al., 1970) estão agregados à zona rural do município, incluindo três lo- calidades: Anel, Salabongá e São Francisco; enquanto os dados coletados em 1991 naquela região são específicos para a vila Anel.

\section{Estado do Rio Grande do Norte}

TABELA 4. Prevalência da Infecção Esquistossomótica e a Cobertura Medicamentosa. Município de Pureza, Rio Grande do Norte, 1977-1989

\begin{tabular}{|c|c|c|c|c|c|c|c|c|c|c|c|c|}
\hline Município & 77 & 78 & 79 & 80 & 81 & 82 & 84 & 85 & 86 & 87 & 88 & 89 \\
\hline \multicolumn{13}{|l|}{ - Pureza } \\
\hline Prevalência & 11,9 & 11,0 & 8,8 & 4,1 & 4,0 & 9,5 & 23,1 & 17,6 & 12,7 & 19,0 & 25,5 & 3,7 \\
\hline \% População tratada & 93,1 & 89,9 & 93,4 & 92,5 & 87,7 & 97,3 & 95,0 & 93,4 & 94,8 & 93,5 & 100,0 & 100,0 \\
\hline
\end{tabular}

Fonte: Fundação Nacional de Saúde. Diretoria Regional do Rio Grande do Norte. / Nota: não houve avaliação no ano de 1983.

TABELA 5. Prevalênica da Infecção e da Forma Hepatosplênica da Esquistossomose, por Grupo Etário e Sexo, na Zona Rural do Município de Pureza, Rio Grande do Norte, 1967 e 1991

\begin{tabular}{|c|c|c|c|c|c|c|c|c|c|c|c|c|c|c|c|c|}
\hline \multirow{2}{*}{$\begin{array}{l}\text { Ano Prevalência } \\
\text { Forma HE. }\end{array}$} & & \multirow[b]{2}{*}{$\mathrm{T}$} & \multirow[b]{2}{*}{ M } & \multirow[b]{2}{*}{$\mathrm{F}$} & \multirow[b]{2}{*}{$\mathrm{T}$} & \multirow[b]{2}{*}{ M } & \multirow[b]{2}{*}{$\mathrm{F}$} & \multirow[b]{2}{*}{$\mathrm{T}$} & \multirow[b]{2}{*}{$\mathrm{M}$} & \multirow[b]{2}{*}{$\mathrm{F}$} & \multirow[b]{2}{*}{$\mathrm{T}$} & \multirow[b]{2}{*}{ M } & \multirow[b]{2}{*}{$\mathrm{F}$} & \multirow[b]{2}{*}{$\mathrm{T}$} & \multirow[b]{2}{*}{ M } & \multirow[b]{2}{*}{$\mathrm{F}$} \\
\hline & & & & & & & & & & & & & & & & \\
\hline \multicolumn{17}{|l|}{$1967^{\mathrm{A}}$} \\
\hline População Examinada & & 599 & 298 & 301 & 178 & 83 & 95 & 130 & 64 & 66 & 156 & 78 & 78 & 135 & 73 & 62 \\
\hline \multirow[t]{2}{*}{ Forma H.E. } & $\mathrm{n}^{\circ}$ & 2,6 & 13 & 13 & 1 & 1 & 0 & 7 & 4 & 3 & 12 & 7 & 5 & 6 & 1 & 5 \\
\hline & $\%$ & 4 & 4 & 4 & 1 & 2 & - & 5 & 6 & 4 & 8 & 9 & 6 & 4 & 1 & 8 \\
\hline \multicolumn{17}{|l|}{$1991^{\mathrm{B}}$} \\
\hline População examinada & & 363 & 184 & 179 & 121 & 59 & 62 & 82 & 41 & 41 & 70 & 36 & 34 & 90 & 48 & 42 \\
\hline \multirow[t]{2}{*}{ Forma H.E. } & $\mathrm{n}^{\circ}$ & 5 & 3 & 2 & o & o & o & o & o & 0 & o & 0 & $\mathrm{O}$ & 5 & $3^{\mathrm{C}}$ & 2 \\
\hline & $\%$ & 1,4 & 1,6 & 1,1 & - & - & - & - & - & - & - & - & - & 5,6 & 6,3 & 4,8 \\
\hline Prevalência & $\%$ & 16,3 & 18,5 & 14,0 & 13,2 & 20,3 & 6,5 & 20,7 & 19,5 & 22,0 & 14,3 & 16,7 & 11,8 & 17,8 & 16,7 & 19,0 \\
\hline
\end{tabular}

A. Fonte: Barbosa et al., (1970b). O ano de 1967 inclui 7 sítios (Jardim, Maceió, Porteirinha, Boa Vista, S. José Tapera, Morada Nova e Olho d'Água).

B. Dados primários. O ano de 1991 inclui apenas 4 dos sítios acima (Jardim, Maceió, S. José Tapera e Olho d’Água

C. Incluindo um caso, 51 anos, esplenectomizado em Natal, 1989. 


\section{Estado de Pernambuco}

Os dados obtidos da antiga Sucam foram limitados. Para o município de Catende, como um todo, as prevalências foram as que se seguem (Pece):

Prevalências

1977/78 Levantamento inicial . . . .62,0\%

$1979 \quad 1^{\underline{a}}$ Avaliação e tratamento . . 30,0\%

$1980 \quad 2^{\underline{a}}$ Avaliação e tratamento . . 18,6\%

$1985 \quad 3^{\mathrm{a}}$ Avaliação e tratamento . . 48,9\%

1990 4 4 Avaliação e tratamento . . 46,5\%
Para a zona rural do município de Catende (1977) existem dados publicados: a prevalência foi de $44,5 \%$, não havendo distribuição por grupos de idade: as formas hepatosplênicas (H.E.) são apresentadas na Tabela 6 .

Dos arquivos do Centro de Pesquisas Aggeu Magalhães constam dados por localidade examinada, embora a distribuição por grupos de idade esteja limitada às crianças/adolescentes do engenho Pau do Óleo (Tabela 7).

Em 1977, as prevalências da infecção, para a população geral, foram as seguintes: engenho Boa Vista 43,4\% e engenho Pau do Óleo 67,5\%. Para o grupo etário 1 - 14 anos a prevalência em 1977 foi de $12,7 \%$ e de 16,7 no grupo de 5 a 14 anos.

TABELA 6. Prevalência das Formas Hepatosplênicas da Esquistossomose em Trabalhadores de Cana nos Engenhos Boa Vista e Pau do Óleo, Município de Catende, Pernambuco, 1977

\begin{tabular}{lccc}
\hline \hline & & \multicolumn{2}{c}{ Forma H.E. } \\
\cline { 3 - 4 } Engenho & Total de & $\mathrm{n}^{\mathbf{0}}$ & $\%$ \\
\hline Boa Vista & Exames Clínicos & & 4,1 \\
Pau do óleo & 121 & $5^{\mathrm{A}}$ & 11,6 \\
\hline \hline
\end{tabular}

Fonte: Costa \& Barbosa, 1980.

A. Incluindo um esplenectomizado.

TABELA 7. Prevalência das Formas Hepatosplênicas da Esquistossomose em Crianças e Adolescentes do Engenho Pau do Óleo, Município de Catende, Pernambuco, 1977. Dados Não Publicados

\begin{tabular}{lccc}
\hline \hline & & \multicolumn{2}{c}{ Forma H.E. } \\
\cline { 3 - 4 } & Total de & $\mathrm{n}^{\underline{0}}$ & $\%$ \\
Grupo Etário & Exames Clínicos & & - \\
\hline $1-4$ & 24 & 0 & 9,1 \\
$10-9$ & 44 & 4 & 26,5 \\
\hline \hline
\end{tabular}

Fonte: Centro de Pesquisas Aggeu Magalhães.

Dados primários para os dois engenhos são apresentados na Tabela 8. 
TABELA 8. Prevalência da Infecção e da Forma Hepatosplência da Esquistossomose, por Grupo Etário e Sexo, nos Engenhos Boa Vista e Pau do Óleo, Município de Catende, Pernambuco, 1991. Dados Primários

\begin{tabular}{|c|c|c|c|c|c|c|c|c|c|c|c|c|c|c|c|c|}
\hline $\begin{array}{l}\text { Ano Prevalência } \\
\text { Forma H.E. }\end{array}$ & & $\mathrm{T}$ & M & $\mathrm{F}$ & $\mathrm{T}$ & M & $\mathrm{F}$ & $\mathrm{T}$ & M & $\mathrm{F}$ & $\mathrm{T}$ & M & $\mathrm{F}$ & $\mathrm{T}$ & M & $\mathrm{F}$ \\
\hline \multicolumn{17}{|l|}{ - Boa Vista } \\
\hline População Examinada & & 327 & 171 & 156 & 117 & 61 & 56 & 87 & 48 & 39 & 69 & 35 & 34 & 54 & 27 & 27 \\
\hline \multirow[t]{2}{*}{ Forma H.E. } & $\mathrm{n}^{\mathrm{o}}$ & 1 & 0 & 1 & 0 & 0 & 0 & 0 & 0 & 0 & 0 & 0 & 0 & 1 & 0 & $1^{\mathrm{A}}$ \\
\hline & $\%$ & 0,3 & - & 0,6 & - & - & - & - & - & - & - & - & - & 1,9 & - & 3,7 \\
\hline Prevalência & $\%$ & 17,1 & 14,0 & 20,5 & 11,9 & 13,1 & 10,7 & 20,3 & 12,5 & 28,2 & 18,8 & 17,1 & 20,6 & 22,2 & 14,8 & 29,6 \\
\hline \multicolumn{17}{|l|}{ - Pau do óleo } \\
\hline População examinada & & 246 & 118 & 128 & 80 & 38 & 42 & 74 & 37 & 37 & 55 & 21 & 34 & 36 & 18 & 18 \\
\hline \multirow[t]{2}{*}{ Fama H.E. } & $\mathrm{n}^{\mathrm{o}}$ & 9 & 4 & 5 & 0 & 0 & 0 & 0 & 0 & 0 & 6 & 2 & $4^{\mathrm{B}}$ & $3^{\mathrm{C}}$ & 2 & 0 \\
\hline & $\%$ & 3,7 & 3,4 & 3,9 & - & - & - & - & - & - & 10,9 & 9,5 & 11,8 & 8,3 & 11,1 & - \\
\hline Prevalência & $\%$ & 47,6 & 46,7 & 48,4 & 42,5 & 39,5 & 45,2 & 56,8 & 62,1 & 51,3 & 45,5 & 47,6 & 44,1 & 41,7 & 33,3 & 50,0 \\
\hline
\end{tabular}

A. Esplenectomisada, 41 anos, no hospital da FSESP em Palmares, Pernambuco, 1989.

B. Uma esplenectomizada, 30 anos, no hospital da FSESP em Palmares, 1987.

C. Um esplenectomizado, 50 anos, no hospital da FSESP em Palmares, 1989.

\section{COMENTÁRIOS E CONCLUSÕES}

As formas H.E. constituem uma das mais graves conseqüências da evolução clínica da esquistossomose. Elas se iniciam na adolescência, caracterizando quadro clínico muito grave que, quando não tratadas, levam geralmente à incapacitação total ou parcial, e comumente ao êxito letal.

Consideradas, há alguns anos, como irreversíveis, as formas H.E. e suas lesões histopatológicas, podem, em alguns casos, regredir "espontaneamente" ou por ação medicamentosa (Bina \& Prata, 1983; Coutinho \& Domingues, 1991; Andrade \& Peixoto, 1992).

O tratamento específico no controle da morbidade da esquistossomose foi revisto com bastante propriedade e abrangência por vários autores, entre eles, Katz (1980), Coura (1984), Coutinho \& Domingues (1991), Gryseels (1992). Este assunto não será discutido no presente trabalho, fazendo-se, entretanto, referências a duas monografias recentemente publicadas (Chen \& Mott, 1988; WHO, 1993).

Com os dados disponíveis ao presente trabalho impõe-se admitir que nenhuma inferência estatística pode ser estabelecida para comparar populações totais em intervalos de tempo longos (14 a 25 anos).
As Tabelas apresentadas para Alagoas mostram que a prevalência da endemia foi substancialmente reduzida (Tabela 1) no período indicado. Em relação às prevalências das formas H.E., em Cajueiro (Tabela 2) e, principalmente, em Viçosa (Tabela 3) mostram redução e, mais do que isso, deslocamento das prevalências para os grupos etários mais velhos, em 1991.

Os resultados para Estado do Rio Grande do Norte indicam, para o município de Pureza, onde o tratamento foi feito anualmente, que as prevalências variaram inexplicavelmente, apesar da elevada cobertura da população medicada (Tabela 4). As formas H.E. comportaram-se de modo semelhante ao Estado de Alagoas, com redução e deslocamento das prevalências para os grupos mais idosos (Tabela 5).

Para o Estado de Pernambuco, a prevalência mostra alguma redução, enquanto os dados sobre as formas H.E. são mais heterogêneas. Os engenhos pesquisados em 1977 (Tabela 6 e 7), no município de Catende, mostram taxas extremamente elevadas para as formas hepatosplências, particularmente nos grupos etários de 5-9 e 10-14 anos (Tabela 7). Em 1991 os dados coletados mostram que as formas H. E. concentravam-se agora, como nos outros estados, nos grupos mais idosos. 
Os resultados deste trabalho, apesar da heterogeneidade de seus dados, sugerem que as prevalências da esquistossomose em áreas rurais selecionadas em três estados nordestinos vêm sofrendo alterações ao longo dos últimos anos. Em Alagoas a redução da prevalência é nítida pelo menos até o ano de 1983. O mesmo declínio foi observado nas localidades estudadas no Rio Grande do Norte e em Pernambuco, ainda que com menos clareza.

O fato que aparece mais constante, em todas as localidades, é o deslocamento das formas H.E. para os grupos mais idosos das populações estudadas no ano de 1991, já observados por outros autores (Coura, 1984; Kloetzel \& Schuster, 1987; Coutinho \& Domingues, 1991).

O presente estudo é limitado por motivos mencionados anteriormente e, conseqüentemente, seus resultados não podem ser generalizados para outras áreas. De qualquer maneira os dados do presente trabalho juntam mais uma limitada contribuição a assunto que vem sendo discutido há alguns anos por numerosos autores, desde que Kloetzel $(1962,1967)$ demonstrou a ação da terapêutica sobre a prevenção das formas H.E. Coutinho \& Domingues (1991) chamam atenção para o trabalho pioneiro de Sette (1953) em Pernambuco, valendo não esquecer as importantes contribuições de Bina (1981) e Bina \& Prata (1983).

Finalmente, vale comentar que não se pode atribuir a possível redução das formas H.E. exclusivamente à ação medicamentosa, já que, como mencionado anteriormente, este fenômeno pode ocorrer "espontaneamente".

Deve-se evitar o excesso de otimismo que alguns estudos acima citados produziram nos meios científicos nacionais e internacionais e que foram estimulados por organizações com interesses diversos, afirmando-se que o problema do controle da esquistossomose estava resolvido. Não se deve também aceitar novas estratégias reducionistas visando especificamente à eliminação das formas hepatosplênicas. Novas alternativas para o controle da esquistossomose vêm sendo discutidas recentemente por Kloetzel \& Schuster (1987), Kloetzel (1989) e Barbosa (1993).

\section{AGRADECIMENTOS}

Aos Dr. Lelis, Chefe do Programa de controle da Esquistossomose da Fundação Nacional de Saúde do Estado do Rio Grande do Norte, e Dr. Ubiracy Guida, Gerente de Endemias da F.N.S. do Estado de Pernambuco, os autores agradecem o fornecimento dos dados secundários utilizados neste trabalho.

\section{RESUMO}

BARBOSA, F. S.; GONÇALVES, J. F. \& MELO, M. C. V. Formas Hepatosplênicas da Esquistossomose Mansônica no Interior do Nordeste do Brasil. Cad. Saúde Públ., Rio de Janeiro, 11 (2): 325-331, abr/jun, 1995.

Estudos de campo sobre a morbidade da esquistossomose mansônica foram realizados em algumas áreas rurais do interior do nordeste do Brasil durante o primeiro semestre de 1991. Estes estudos seccionais foram limitados à determinação do gradiente clinico da endemia. Foram examinadas todas as populações presentes neste levantamento feito casa-por-casa. Os resultados mostraram a redução da prevalência da forma mais grave da esquistossomose - a forma hepatosplênica quando comparados com estudos semelhantes feitos nas mesmas áreas durante as décadas de 60 e 70. As taxas de prevalência das formas clínicas graves da doença foram deslocadas dos grupos de idade mais jovens para os grupos mais velhos.

Palavras-Chave: Esquistossomose; Morbidade; Epidemiologia

\section{REFERÊNCIAS BIBLIOGRÁFICAS}

ANDRADE, Z. \& PEIXOTO, E.,1992. Pathology of periportal fibrosis involution in human schistosomiasis. Revista do Instituto de Medicina Tropical de São Paulo, 34:263-272.

BARBOSA, F. S., 1965. Morbidade na esquistossomose. Estudo em quatro localidades do Estado de Pernambuco. Revista Brasileira de Malariologia e Doenças Tropicais, 18: 153-159. 
1993. Socio-economic Components and Community Participation in the Determination and Control of Schistosomiasis. $4^{\text {th }}$ International Symposium on Schistosomiasis, november 29, Rio de Janeiro, Brasil. (Mimeo.)

BARBOSA, F. S.; COSTA, D.; PINTO, R. F.; BARBOSA, J. M. \& RODRIGUES, B. A., 1970a Levantamentos seccionais sobre a esquistossomose no nordeste do Brasil. I. Estado de Alagoas. Revista da Sociedade Brasileira de Medicina Tropical, 4: 129-132.

BARBOSA, F. S.; PESSOA, D.; OLIVEIRA, P.; BARBOSA, J. M. \& RODRIGUES, B. A., 1970b. Levantamentos seccionais sobre a esquistossomose no nordeste do Brasil. II. Estado do Rio Grande do Norte. Revista da Sociedade Brasileira de Medicina Tropical, 4: 195-197.

BARBOSA, F. S. \& VOSS, H., 1969. Evolution of the clinical gradient of schistosomiasis mansoni infection in a small town in northeastern Brazil. Bulletin of the World Health Organization, 40: 966-969.

BINA, J. C., 1981. Influência da terapêutica específica na evolução da esquistossomose mansoni. Revista de Patologia Tropical, 10: 221-267.

BINA, J. \& PRATA, A., 1983. Regressão da hepatosplenomegalia pelo tratamento específico da esquistossomose. Revista da Sociedade Brasileira de Medicina Tropical, 16: 213-218.

BRENER, Z. \& MOURÃO, O. G., 1956. Inquéritos clínico-epidemiológicos em focos endêmicos da esquistossomose mansoni em Minas Gerais. Revista Brasileira de Malariologia e Doenças Tropicais, 8: 519-526.

CHEN, M. G. \& MOTT, K. E., 1988. Progress in assessment of morbidity due to Schistosoma mansoni infection. In: Progress in Assement of Morbidity Due to Schistosomiasis, R2-R40, London: Bureau of Hygiene and Tropical Diseases.

COSTA, D. P. P. \& BARBOSA, F. S., 1980. Esquistossomose em trabalhadores da Usina Catende, Pernambuco, Brasil. Revista de Saúde Pública, 14: 469-474.

COURA, J. R., 1984. Morbidade da esquistossomose mansoni no Brasil. III. Estudo evolutivo em uma área endêmica no período de dez anos. Memórias do Instituto Oswaldo Cruz, 79: 447-453.

COUTINHO, A. \& DOMINGUES, A. L. C., 1991. O tratamento específico no controle da morbidade. In: Tópicos em Gastroenterologia, pp. 141-159, Rio de Janeiro: MEDSI. (Gastroenterologia Tropical, 2)
GRYSEELS, B., 1992. Morbidity due to infection with Schistosoma mansoni: an update. Tropical and Geographical Medicine, 44: 189-200.

KATZ, N., 1980. Experiências com quimioterapia em grande escala no controle da esquistossomose no Brasil. Revista do Instituto de Medicina Tropical de São Paulo, 22: 40-52.

KATZ, N. \& BRENER, Z., 1966. Evolução de 112 casos de esquistossomose mansoni observados após 10 anos de permanência em focos endêmicos de Minas Gerais. Revista do Instituto de Medicina Tropical de São Paulo, 8: 139-142.

KLOETZEL, K.,1962. Aspectos Epidemiológicos da Esquistossomose Mansônica em uma População de Pernambuco. Tese de Livre Docência, São Paulo: Universidade de São Paulo.

1967. A suggestion for the prevention of severe clinical forms of schistosomiasis mansoni. Bulletin of the World Health Organization, 37: 686-687.

1989. Schistosomiasis in Brazil: Does social development suffice? Parasitology Today, 5: 388-391.

KLOETZEL, K. \& SCHUSTER, N. H., 1987. Repeated mass treatment of schistosomiasis mansoni: Experience in hyperendentic areas of Brazil. I. Parasitological effects and morbidity. Transactions of the Royal Society of Tropical Medicine and Hygiene, 81: 365-370.

PESSOA, S. B. \& AMORIM, J. P., 1957. Notas sobre a esquistossomose mansônica em algumas localidades de Alagoas. Revista Brasileira de Medicina, 14: 420-422.

PESSOA, S. B. \& BARROS, P. R., 1953. Notas sobre a epidemiologia da esquistossomose mansônica no Estado de Sergipe. Revista de Medicina e Cirurgia de São Paulo, 13: 147-154.

PESSOA, S. B.; SILVA, L. M. P. \& COSTA, L., 1955. Observações sobre a epidemiologia da esquistossomose no Estado da Paraíba. Revista Brasileira de Malariologia e Doenças Tropicais, 7: 305-310.

WHO (World Health Organizadon), 1993. The Control of Schistosomiasis. Second Report of the WHO Expert Committee. Geneva: WHO. 